University of Wollongong

Research Online

Australian Institute for Innovative Materials -

Papers

Australian Institute for Innovative Materials

2013

Investigation of the critical behavior in Mn0.94Nb 0.06CoGe alloy by using the field dependence of magnetic entropy change

J C. Debnath

University Of Wollongong, jcd341@uow.edu.au

P Shamba

University of Wollongong, ps807@uowmail.edu.au

A M. Strydom

University of Johannesburg

Jianli Wang

University of Wollongong, jianli@uow.edu.au

S X. Dou

University of Wollongong, shi@uow.edu.au

Follow this and additional works at: https://ro.uow.edu.au/aiimpapers

Part of the Engineering Commons, and the Physical Sciences and Mathematics Commons

Research Online is the open access institutional repository for the University of Wollongong. For further information contact the UOW Library: research-pubs@uow.edu.au 


\title{
Investigation of the critical behavior in Mn0.94Nb 0.06CoGe alloy by using the field dependence of magnetic entropy change
}

\begin{abstract}
The critical behaviour of Mn0.94Nb0.06CoGe alloy around the paramagnetic-ferromagnetic phase transition was studied based on the field dependence on magnetic entropy change. By using the obtained exponents, the modified Arrott plot is consistent with that by using conventional method. These critical exponents are confirmed by the Widom scaling relation. Based on these critical exponents, the magnetization, field and temperature data around Tc collapse into two curves obeying the single scaling equation $\mathrm{M} \partial \mathrm{H} ; \mathrm{e})^{1 / 4} \mathrm{ebf6}(\mathrm{H} / \mathrm{ebpc})$. The calculated critical exponents not only obey the scaling theory but also anastomose the deduced results from the Kouvel-Fisher method [J. S. Kouvel and M. E. Fisher, Phys. Rev. 136, A1626 (1964)]. The values deduced for the critical exponents in the Mn0.94Nb0.06CoGe alloy are close to the theoretical prediction of the mean-field model, indicating that the magnetic interactions are long range. This method eliminates the drawback due to utilization of multistep nonlinear fitting in a conventional manner. So it provides an alternative method to investigate the critical behaviour.
\end{abstract}

\section{Keywords}

entropy, magnetic, dependence, field, alloy, 06coge, 94nb, mn0, change, behavior, investigation, critical

Disciplines

Engineering | Physical Sciences and Mathematics

\section{Publication Details}

Debnath, J. C., Shamba, P., Strydom, A. M., Wang, J. L. \& Dou, S. X. (2013). Investigation of the critical behavior in $\mathrm{Mn} 0.94 \mathrm{Nb} 0.06 \mathrm{CoGe}$ alloy by using the field dependence of magnetic entropy change. Journal of Applied Physics, 113 (9), 093902-1-093902-5. 


\title{
AIP Appilied Physics
}

\section{Investigation of the critical behavior in Mn0.94Nb0.06CoGe alloy by using the field dependence of magnetic entropy change}

\author{
J. C. Debnath, P. Shamba, A. M. Strydom, J. L. Wang, and S. X. Dou
}

Citation: J. Appl. Phys. 113, 093902 (2013); doi: 10.1063/1.4794100

View online: http://dx.doi.org/10.1063/1.4794100

View Table of Contents: http://jap.aip.org/resource/1/JAPIAU/v113/i9

Published by the American Institute of Physics.

\section{Related Articles}

Magnetic properties of the martensitic phase in Ni-Mn-In-Co metamagnetic shape memory alloys Appl. Phys. Lett. 102, 101908 (2013)

Field-induced magnetic phase transition in $\mathrm{Pr} 3+$ doped $\mathrm{Sm} 0.5 \mathrm{Sr} 0.5 \mathrm{MnO} 3$ manganites J. Appl. Phys. 113, 17D706 (2013)

Effects of carbon content on structural, magnetic, and electrical/thermal transport properties of antiperovskite compounds GaCxFe3

J. Appl. Phys. 113, 103906 (2013)

Freezing of the octahedral tilt near ferromagnetic transition and appearance of a glassy phase at low temperature driven by the tilt instabilities in $\mathrm{SrRuO3}$

J. Appl. Phys. 113, 17E122 (2013)

Investigation of spin ordering in antiferromagnetic Fe1-xMnxPO4 with Mössbauer spectroscopy

J. Appl. Phys. 113, 17E121 (2013)

\section{Additional information on J. Appl. Phys.}

Journal Homepage: http://jap.aip.org/

Journal Information: http://jap.aip.org/about/about_the_journal

Top downloads: http://jap.aip.org/features/most_downloaded

Information for Authors: http://jap.aip.org/authors

\section{ADVERTISEMENT}

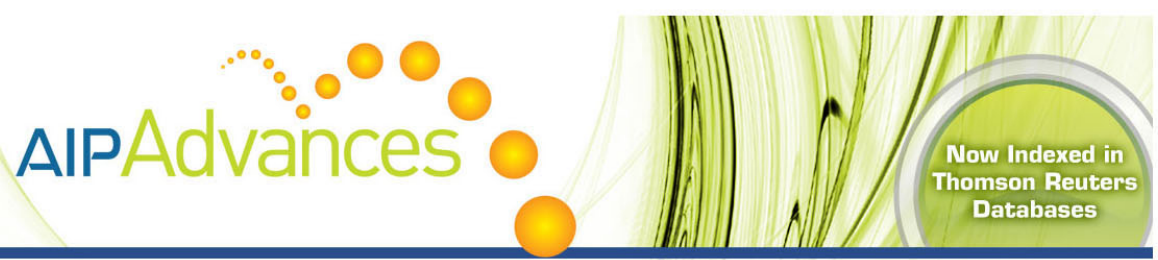

\section{Explore AIP's open access journal: Rapid publication \\ - Article-level metrics \\ Post-publication rating and commenting}




\title{
Investigation of the critical behavior in $\mathrm{Mn}_{0.94} \mathrm{Nb}_{0.06}$ CoGe alloy by using the field dependence of magnetic entropy change
}

\author{
J. C. Debnath, ${ }^{1, \text { a) }}$ P. Shamba, ${ }^{2}$ A. M. Strydom, ${ }^{1}$ J. L. Wang, ${ }^{2,3}$ and S. X. Dou ${ }^{2}$ \\ ${ }^{1}$ Department of Physics, University of Johannesburg, P.O. Box 524, Auckland Park 2006, South Africa \\ ${ }^{2}$ Institute for Superconducting and Electronic Materials, University of Wollongong, Squaire Way, \\ North Wollongong, Wollongong, NSW 2500, Australia \\ ${ }^{3}$ Bragg Institute, Australian Nuclear Science and Technology Organization, Lucas Heights, NSW 2234, \\ Australia.
}

(Received 1 February 2013; accepted 19 February 2013; published online 1 March 2013)

\begin{abstract}
The critical behaviour of $\mathrm{Mn}_{0.94} \mathrm{Nb}_{0.06} \mathrm{CoGe}$ alloy around the paramagnetic-ferromagnetic phase transition was studied based on the field dependence on magnetic entropy change. By using the obtained exponents, the modified Arrott plot is consistent with that by using conventional method. These critical exponents are confirmed by the Widom scaling relation. Based on these critical exponents, the magnetization, field and temperature data around Tc collapse into two curves obeying the single scaling equation $M(H, \varepsilon)=\varepsilon^{\beta} f \pm\left(H / \varepsilon^{\beta+\gamma}\right)$. The calculated critical exponents not only obey the scaling theory but also anastomose the deduced results from the Kouvel-Fisher method [J. S. Kouvel and M. E. Fisher, Phys. Rev. 136, A1626 (1964)]. The values deduced for the critical exponents in the $\mathrm{Mn}_{0.94} \mathrm{Nb}_{0.06} \mathrm{CoGe}$ alloy are close to the theoretical prediction of the mean-field model, indicating that the magnetic interactions are long range. This method eliminates the drawback due to utilization of multistep nonlinear fitting in a conventional manner. So it provides an alternative method to investigate the critical behaviour. (C) 2013 American Institute of Physics. [http://dx.doi.org/10.1063/1.4794100]
\end{abstract}

\section{INTRODUCTION}

The magnetocaloric effect (MCE) has attracted much attention for its potential application in magnetic refrigeration. ${ }^{1,2}$ The MCE has been observed in two kinds of magnetic materials: those possessing a first order magnetic transition (FOMT) and those possessing a second-order magnetic transition (SOMT). It became clear that materials undergoing a FOMT exhibit a large MCE because of the strong coupling between magnetism and structure in these materials. Typical representative materials include $\mathrm{Gd}_{5}\left(\mathrm{Si}_{1-\mathrm{x}} \mathrm{Ge}_{\mathrm{x}}\right)_{4},{ }^{3} \mathrm{MnFeP}_{1-\mathrm{x}} \mathrm{As}_{\mathrm{x}},{ }^{4} \mathrm{La}\left(\mathrm{Fe}_{1-\mathrm{x}} \mathrm{Si}_{\mathrm{x}}\right)_{13},{ }^{5} \mathrm{LaFe}_{11.7}$ $\mathrm{Si}_{1.3} \mathrm{H}_{x},{ }^{6} \mathrm{MnAs}_{1-\mathrm{x}} \mathrm{Sb}_{\mathrm{x}},{ }^{7}$ and Ni-Mn-Ga. ${ }^{8}$ Perovskite manganites are also considered to be promising candidate for the magnetic refrigeration. ${ }^{9-11}$ Since the magnetic properties of perovskite manganites, Curie temperature and saturation magnetization, are strongly doping-dependent, these typical materials are believed to be good candidates for magnetic refrigeration at various temperatures. ${ }^{12,13}$ However, for different reasons, none of these materials have yet been employed in a commercial refrigerator. Obviously, there is a need for yet further improved materials.

Recently, a lot of research interest has been paid to the MnCoGe material system for use as a magnetic refrigerant. ${ }^{14-16}$ This material system undergoes a second order phase transition as well as a crystallographic phase transition from the low temperature orthorhombic TiNiSi type to the high temperature hexagonal $\mathrm{Ni}_{2}$ In type structure. ${ }^{17}$ Although different properties of $\mathrm{MnCoGe}$ have been extensively investigated, more studies are desired to understand the intrinsic magnetic

\footnotetext{
a) Author to whom correspondence should be addressed. Electronic addresses: jcd341@uowmail.edu.au and jdebnath@uj.ac.za.
}

interactions. Recent investigations have revealed that an effective way of probing the magnetic interactions responsible for the magnetic transitions is by performing a critical exponent analysis in the vicinity of the ferromagnetic (FM)-paramagnetic (PM) region. ${ }^{18-20}$ Thus, in an effort to understand the nature of the magnetic transition in $\mathrm{Mn}_{0.94} \mathrm{Nb}_{0.06} \mathrm{CoGe}$, we performed a critical exponent study by using the field dependence of magnetic entropy change method. To date, to the best of our knowledge, no experimental study of the critical phenomena has been reported for the $\mathrm{Mn}_{1-\mathrm{x}} \mathrm{Nb}_{\mathrm{x}} \mathrm{CoGe}$ system by using the above mentioned method.

Generally, the common method to deduce the critical exponents always depends on the fitting values obtained from the Arrott plot according to Arrott-Noakes equation of state, $(H / M)^{1 / \gamma}=\left(T-T_{C}\right) / T_{C+}\left(M / M_{1}\right)^{1 / \beta},{ }^{21}$ where $\mathrm{M}_{1}$ is a material constant, and $\beta$ and $\gamma$ are the critical exponents. Due to the unknown accurate critical exponents at the beginning of work, the researcher has to apply some different theoretical models to first construct some tentative Arrott plots and then choose the best one for fitting data. ${ }^{22-24}$ However, the choice of model and fitting range will directly determine the finial critical values. Different researchers make different choices. Therefore, a considerable uncertainty is unavoidable.

To eliminate the drawback, instead, we use the field dependence of magnetic entropy change to deduce the critical exponents. Due to the inner correlation between the critical exponents, they can be calculated from the related equation set. Moreover, in this letter, the calculated exponents not only agree with those deduced from Kouvel-Fisher (KF) method but also obey the scaling theory, ${ }^{25,26}$ indicating that the obtained critical exponents are reliable. Therefore, this method can be widely applied to the similar research field. 
In this paper, we focus on the magnetic properties and critical behaviour of the $\mathrm{Mn}_{0.94} \mathrm{Nb}_{0.06} \mathrm{CoGe}$ alloy. Thus in an effort to understand the nature of the magnetic transition in $\mathrm{Mn}_{0.94} \mathrm{Nb}_{0.06} \mathrm{CoGe}$, we performed a critical exponent analysis in the vicinity of the FM-PM region. ${ }^{18-20}$ We demonstrate that the critical phenomena near PM-FM Curie temperature transition are described by using the field dependence of magnetic entropy change rather than by other models. The mean field interaction model for long range ordering has theoretical critical exponents of $\beta=0.5$, $\gamma=1.0$, and $\delta=3.0$. The $\delta, \beta$, and $\gamma$ values derived for the $\mathrm{Mn}_{0.94} \mathrm{Nd}_{0.06} \mathrm{CoGe}$ alloy are close to the mean field values. Apart from a slight increase in $\beta$ and decrease in $\gamma$ as well as slight decrease in $\delta$, the deduced critical exponents are very close to the theoretical values of mean-field model, indicating that the existence of the long-range interactions dominates the critical behavior around $\mathrm{T}_{\mathrm{C}}$. Our results reveal that this method can be used to investigate critical behavior and eliminates the drawback due to utilization of multistep nonlinear fitting in a conventional manner.

\section{EXPERIMENTAL DETAILS}

Polycrystalline $\mathrm{Mn}_{0.94} \mathrm{Nb}_{0.06}$ CoGe ingot was prepared by arc melting the appropriate amounts of $\mathrm{Mn}(99.9 \%), \mathrm{Nb}$ (99.999\%) powder, Co (99.9\%), and Ge (99.999\%) chips in an argon atmosphere. During arc melting, a 5\% excess Mn over the stoichiometric amount was added to compensate the weight loss of $\mathrm{Mn}$. The polycrystalline ingot was melted several times to achieve good homogeneity. The ingot was then wrapped in tantalum foil, sealed in a quartz ampoule and subsequently annealed at $850^{\circ} \mathrm{C}$ for $120 \mathrm{~h}$, and then quenched in water at room temperature. The magnetization measurements were carried out using the vibration sample magnetometer option of a Quantum Design 14 T Physical Property Measurement System in the temperature range of 100-320 K at applied fields of up to $5 \mathrm{~T}$. For critical exponent study, magnetization isotherms were measured in the temperature range $247-301 \mathrm{~K}$ with an interval of $2 \mathrm{~K}$ at applied fields of up to $5 \mathrm{~T}$.

\section{RESULT AND DISCUSSION}

Shown in Figure 1(a) is the temperature dependence $[220 \mathrm{~K}$ to $320 \mathrm{~K}]$ of the magnetization $(\mathrm{M}-\mathrm{T})$ of the $\mathrm{Mn}_{0.94} \mathrm{Nb}_{0.06} \mathrm{CoGe}$ alloy measured in a magnetic field of 100 Oe. All these data were taken in the warming run after zero-field cooling (ZFC) and field cooling (FC), respectively. The M-T curve exhibits a sharp PM-FM phase transition. The Curie temperature $\left(\mathrm{T}_{C}\right)$, defined by the minimum in $\mathrm{dM} / \mathrm{dT}$, has been determined to be $T_{C}=268 \mathrm{~K}$ (the inset of Figure 1(a)). The absence of thermal hysteresis in the vicinity of the magnetic PM to FM transition is a clear evidence of the second order nature of this transition. Here, in order to further clarify the nature of the FM-PM phase transition, we measure the isothermal magnetization versus applied field around the Curie temperature, which has been shown in Figure 1(b).

According to the scaling hypothesis, ${ }^{27}$ a second-order magnetic phase transition near Curie point is characterized by a set of critical exponents of $\beta, \gamma$, and $\delta$. In order to deduce these parameters, the isothermal magnetization
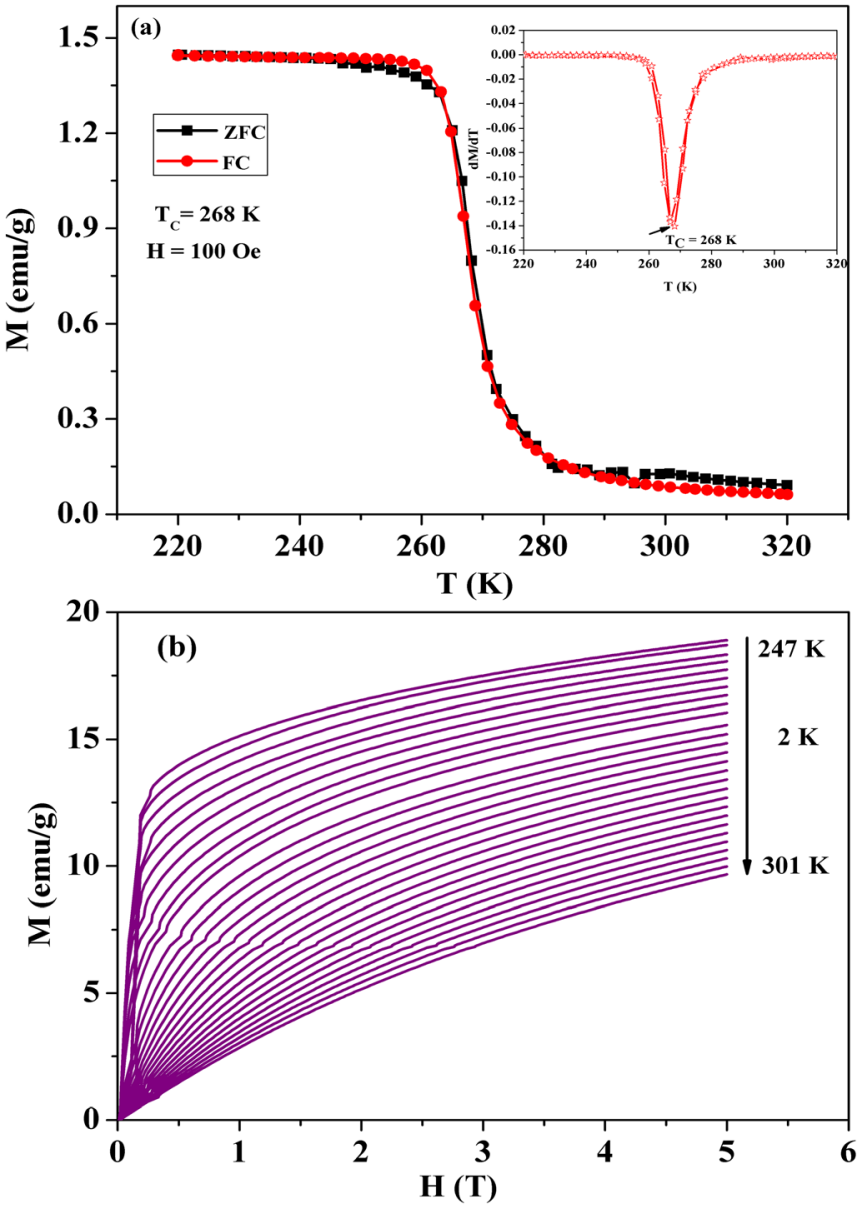

FIG. 1. (a) Temperature dependence of magnetization at $\mathrm{H}=100$ Oe. Inset shows the plot of $\mathrm{dM} / \mathrm{dT}$ vs $\mathrm{T}$. (b) Isothermal magnetization vs magnetic field at different temperatures around $\mathrm{T}_{\mathrm{C}}$.

curves ( $\mathrm{M}$ versus $\mathrm{H}$ ) should be changed into the so-called Arrott plot, namely, $\mathrm{M}^{2}$ versus $\mathrm{H} / \mathrm{M}$. If the system is in line with the Landau mean-field theory, ${ }^{27}$ the relationship of $\mathrm{M}^{2}$ versus $\mathrm{H} / \mathrm{M}$ should be shown as a set of parallel straight lines around $\mathrm{T}_{\mathrm{C}}$. Meanwhile, the order of magnetic transition can be determined from the slope of straight line. ${ }^{28}$

A plot of $\mathrm{H}^{2}$ versus $\mathrm{M}^{2}$, known as the Arrott plot, is shown in Figure 2(a) for the temperatures in the vicinity of $\mathrm{T}_{\mathrm{C}}$. According to the criterion proposed by Banerjee, ${ }^{28}$ the order of magnetic transition can be determined from the slope of the isotherm plot. If all $\mathrm{H}^{2}$ versus $\mathrm{M}^{2}$ curves show a negative slope, the transition is of first order while a positive slope corresponds to a second order transition. For the $\mathrm{Mn}_{0.94} \mathrm{Nb}_{0.06} \mathrm{CoGe}$ alloy, the presence of the positive slope of the $\mathrm{H}^{2}$ versus $\mathrm{M}^{2}$ curves indicates that the phase transition is a second order PM-FM transition. This result is consistent with the absence of thermal hysteresis in the vicinity of the PM-FM transition in Figure 1(a), thereby again confirming the second order nature of this transition.

However, a close inspection of the $\mathrm{H}^{2}$ versus $\mathrm{M}^{2}$ curves reveals that all the curves are not parallel to each other, indicating that the critical components of $\beta=0.5$ and $\gamma=1$ are not satisfied. Namely, the mean-field theory cannot be used to describe the critical behavior in the present system. In order to obtain the accurate critical exponents, the 

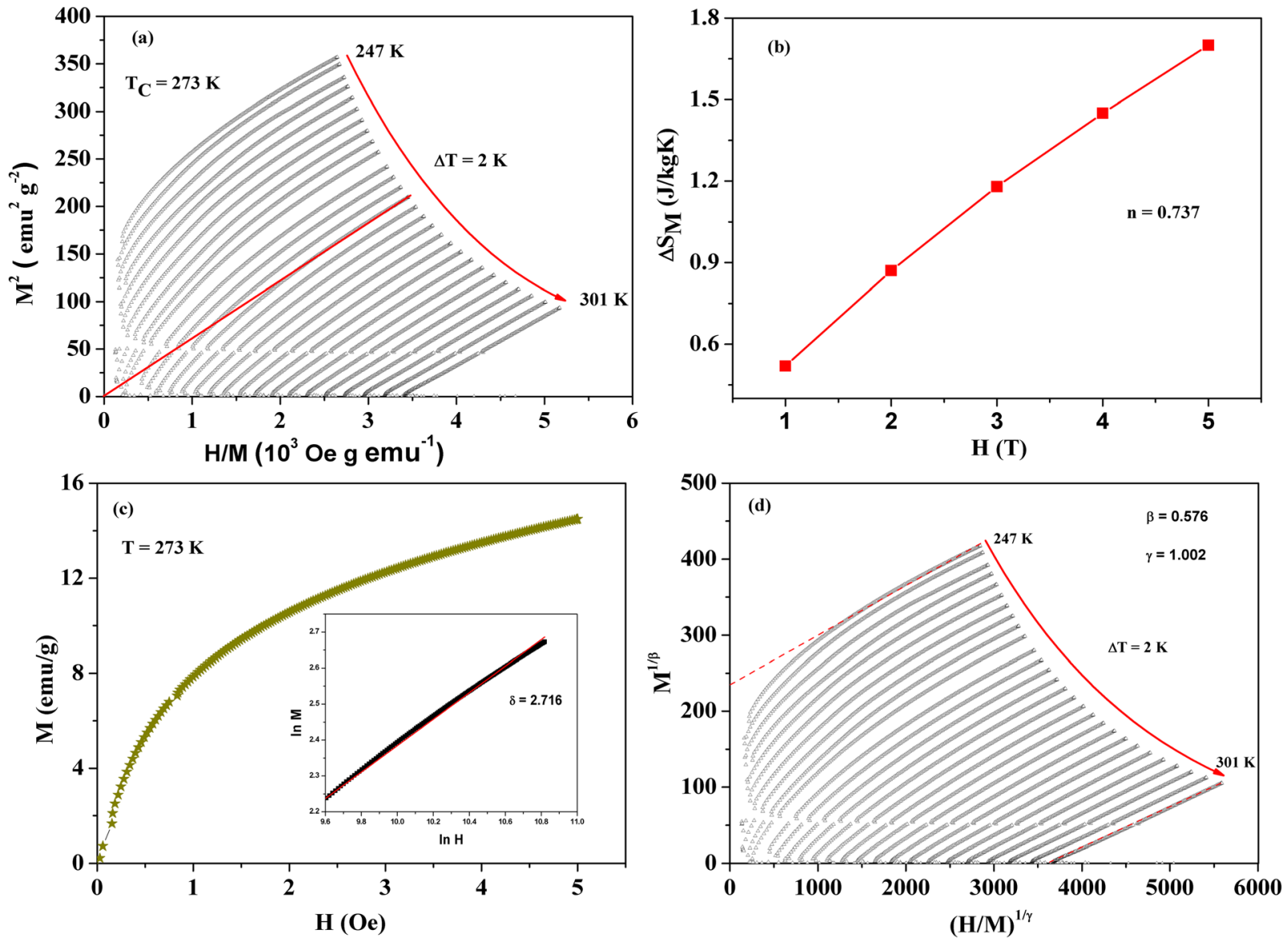

FIG. 2. (a) Arrott plot of $\mathrm{M}^{2}$ vs $\mathrm{H} / \mathrm{M}$ at temperatures in the vicinity of $\mathrm{T}_{\mathrm{C}}$; (b) the maximal entropy change vs applied magnetic field and the solid line is the fitting curve; (c) isotherm M vs $\mathrm{H}$ plot at $273 \mathrm{~K}$; the inset shows the same plot in log-log scale, and solid line is the linear fit following $M_{T_{C}}=D H^{1 / \delta}, \varepsilon=0, T=T_{C} ;\left(\right.$ d) modified Arrott plot: isotherms of $M^{1 / \beta}$ vs $\left(\frac{H}{M}\right)^{1 / \gamma}$ with the calculated $\beta=0.576$ and $\gamma=1.002$.

conventional method is to use some tentative exponents to construct a new Arrott plot and then fit the data of the linear part or directly fit the data of the initial Arrott plot. After that, the obtained intercepts on the $\mathrm{x}(\mathrm{y})$-axis were performed with multistep nonlinear fitting until the final critical exponents reach steady values. Because of the drawback of this method as mentioned above, we adopted another way to deduce the critical exponents. First, these critical exponents of $\beta, \gamma$, and $\delta$ satisfy the Widom scaling relation: $\delta=1+\gamma / \beta{ }^{29}$ Meanwhile, the critical exponent of $\delta$ is associated with the critical magnetization isotherm at $\mathrm{T}_{\mathrm{C}}$ and can be obtained from the following equation: $M_{T_{C}}=D H^{1 / \delta}, \varepsilon=0, T=T_{C}$, where $\mathrm{D}$ is the critical amplitudes. ${ }^{30}$

Notably, we need to establish another equation to solve $\beta$ and $\gamma$. In a magnetic system with a second-order phase transition, Oesterreicher and Parker previously proposed a universal relation of the field dependence of magnetic entropy change, namely, $\left|\Delta \mathrm{S}_{\mathrm{M}}\right| \propto \mathrm{H}^{n}$, where $n=2 / 3 .^{31}$ However, the subsequent experimental results exhibit deviation from $n=2 / 3$ in the soft magnetic amorphous alloys. Recently, Franco et al. further confirmed the existence of the universal relation and reprovided a new relation that agrees better with experimental results ${ }^{32}$

$$
n=1+\frac{\beta-1}{\beta+\gamma} .
$$

This relation has been applied to some different universality classes, such as the soft magnetic $\mathrm{Fe}_{83} \mathrm{Zr}_{6} \mathrm{~B}_{10} \mathrm{Cu}_{1}$ and the cubic Laves phase compounds $\mathrm{TbCo}_{2} \cdot{ }^{33,34}$ Thus, if we can know the value of $n$, the critical exponents of $\beta$ and $\gamma$ will be solved. In order to obtain the value of $n$, we first calculated the magnetic entropy change versus temperature under different magnetic fields using the conventional method. ${ }^{35}$ From it, we obtained the relation of $\left|\Delta \mathrm{S}_{\mathrm{M}}\right|$ versus $\mathrm{H}$, as shown by the red squares in Figure 2(b). Then, using the above function of $\left|\Delta \mathrm{S}_{\mathrm{M}}\right| \alpha \mathrm{H}^{n}$, the value of $n$ was determined to be $n=0.737$. Now, we chose $\mathrm{M}$ versus $\mathrm{H}$ curve at $\mathrm{T}_{\mathrm{C}}$ for calculating $\delta$. As shown in the inset of Figure 2(c), the value of $\delta$ is 2.716. Using the obtained values of $n$ and $\delta$, the values of $\beta$ and $\gamma$ were calculated to be 0.576 and 1.002, respectively. Thus, a new Arrott plot can be redrawn with the obtained $\beta$ and $\gamma$. Using the critical exponents $\beta=0.576$ and $\gamma=1.002$ at $\mathrm{T}=273 \mathrm{~K}$, a new Arrott plot has been drawn in the Figure 2(d). Obviously, a set of parallel lines from 247 to $301 \mathrm{~K}$ exhibit in the plot, indicating that the calculated exponents are the popular results.

The reliability of the calculated exponents $\beta$ and $\gamma$ can be confirmed by using the scaling theory. In the critical region, according to the scaling theory, the magnetic equation of state can be written as

$$
M(H, \varepsilon)=\varepsilon^{\beta} f \pm\left(H / \varepsilon^{\beta+\gamma}\right),
$$


where $\varepsilon$ is the reduced temperature $\left(\mathrm{T}-\mathrm{T}_{\mathrm{C}}\right) / \mathrm{T}_{\mathrm{C}}$ and $f_{+}$and $f_{-}$are regular analytical functions above and below $\mathrm{T}_{\mathrm{C}}$, respectively. Using $\beta$ and $\gamma$ obtained from the field dependence on magnetic entropy change method, the plots of $\mathrm{M} / \varepsilon^{\beta}$ versus $H / \varepsilon^{(\beta+\gamma)}$ as shown in Figure 3(a) yield two universal curves, one for temperatures above $\mathrm{T}_{\mathrm{C}}$ and the other one for temperatures below $\mathrm{T}_{\mathrm{C}}$, in agreement with the scaling theory. Therefore, the FM behavior around the Curie temperature was properly renormalized following the scaling equation of state. This therefore confirms that the obtained values of the critical components as well as the $\mathrm{T}_{\mathrm{C}}$ are reasonably accurate and unambiguous as well as in agreement with the scaling hypothesis. The critical behaviour analysis of the $\mathrm{Mn}_{0.94}$ $\mathrm{Nb}_{0.06} \mathrm{CoGe}$ alloy reveals a magnetic transition at $\mathrm{T}_{\mathrm{C}} \approx 273 \mathrm{~K}$.

In addition, we also used the KF method to deduce the critical exponents for comparison with the critical exponents calculated from field dependence of magnetic entropy change. ${ }^{25}$ The Kouvel-Fisher method which makes use of following equations is a way of determining the critical exponents $\beta$ and $\gamma$

$$
\begin{gathered}
\frac{M_{S}(T)}{d M_{S}(T) / d T}=\frac{T-T_{C}}{\beta}, \\
\frac{\chi_{0}^{-1}(T)}{d \chi_{0}^{-1}(T) d T}=\frac{T-T_{C}}{\gamma} .
\end{gathered}
$$
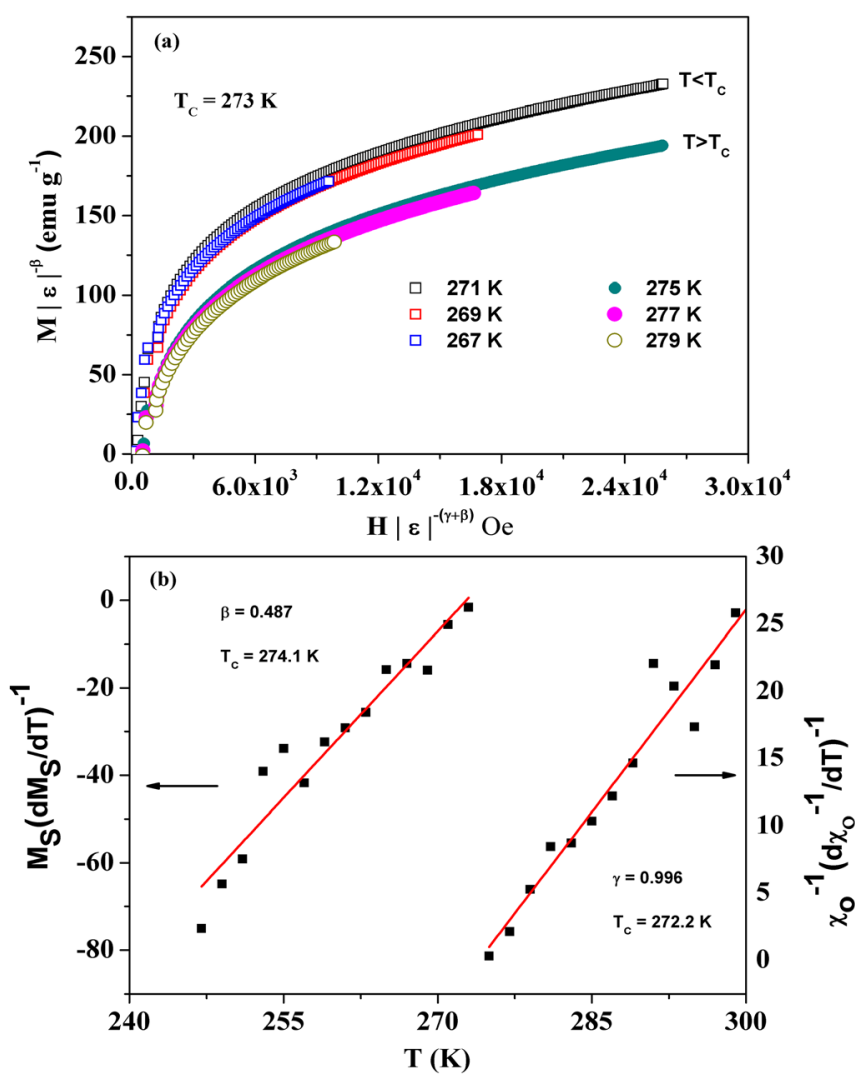

FIG. 3. (a) Scaling plots indicating universal curves below and above $\mathrm{T}_{\mathrm{C}}$ for the $\mathrm{Mn}_{0.94} \mathrm{Nb}_{0.06}$ CoGe alloy using the exponents $\beta=0.576$ and $\gamma=1.002$. (b) Kouvel-Fisher plot for the spontaneous magnetization $M_{S}(T)$ and the inverse initial susceptibility $\chi_{0}^{-1}(\mathrm{~T})$ (solid lines are fitted to Eqs. (3) and (4).
According to these equations, plotting $M_{S}(T)\left[d M_{S} / d T\right]^{-1}$ and $\chi_{0}^{-1}(T)\left[d \chi_{0}^{-1} / d T\right]^{-1}$ versus temperature yields straight lines with slopes of $1 / \beta$ and $1 / \gamma$, respectively. The value of $\mathrm{T}_{\mathrm{C}}$ is obtained from the intercepts on the $x$-axis. As presented in Figure 3(b), the critical exponents $\beta$ and $\gamma$ as well as $\mathrm{T}_{\mathrm{C}}$ obtained using the Kouvel-Fisher method are $\beta=0.487$ $\pm 0.04, \mathrm{~T}_{\mathrm{C}}=274.1 \mathrm{~K}$ and $\gamma=0.996 \pm 0.02, \mathrm{~T}_{\mathrm{C}}=272.2 \mathrm{~K} . \mathrm{A}$ comparison of the critical exponents $\beta$ and $\gamma$ obtained using the field dependence of magnetic entropy change and those obtained using the KF method reveals that these values match reasonably well. Hence, we think that the present calculated method for the study of critical properties is effective and feasible.

The mean field interaction model for long range ordering has theoretical critical exponents of $\beta=0.5, \gamma=1.0$, and $\delta=3.0 .^{32}$ The $\delta, \beta$, and $\gamma$ values derived for the $\mathrm{Mn}_{0.94}$ $\mathrm{Nd}_{0.06} \mathrm{CoGe}$ alloy are close to the mean field values, thus indicating that long range interactions dominate the critical behavior around $\mathrm{T}_{\mathrm{C}}$. It is also speculated that the competition between the localized Mn-Mn magnetic interactions should be responsible for the critical behavior in this system.

\section{CONCLUSIONS}

In this work, we have studied the critical behavior in $\mathrm{Mn}_{0.94} \mathrm{Nb}_{0.06} \mathrm{CoGe}$ alloy by using the field dependence of magnetic entropy change. The validity of the calculated critical exponents was confirmed by the scaling theory. The determined critical exponents are close to those predicted by the mean-field theory, with long range interactions. The simple method effectively avoids the drawback of uncertainty. Therefore, this method can be extensively applied to investigate the critical behavior.

\section{ACKNOWLEDGMENTS}

A.M.S. thanks the NRF-SA (78832) for funding support. J.C.D. acknowledges the FRC and URC of UJ for a Postdoctoral Fellowship, supervised by A.M.S. This work was also supported by an Australian Research Council Discovery Project (DP0879070).

\footnotetext{
${ }^{1}$ A. M. Tishin, in Handbook of Magnetic Materials, edited by K. H. J. Buschow (Elsevier, Amsterdam, 1999), Vol. 12, pp. 395-524.

${ }^{2}$ E. Brück, J. Phys. D: Appl. Phys. 38, 381 (2005).

${ }^{3}$ V. K. Pecharsky and K. A. Gschneidner, Phys. Rev. Lett. 78, 4494 (1997).

${ }^{4}$ O. Tegus, E. Brück, K. H. J. Buschow, and F. R. de Boer, Nature (London) 415, 150 (2002).

${ }^{5}$ F. X. Hu, B. G. Shen, J. R. Sun, Z. H. Cheng, G. H. Rao, and X. X. Zhang, Appl. Phys. Lett. 78, 3675 (2001).

${ }^{6}$ J. C. Debnath, R. Zeng, J. H. Kim, P. Shamba, and S. X. Dou, Appl. Phys. A 106, 245 (2012).

${ }^{7}$ H. Wada and Y. Tanabe, Appl. Phys. Lett. 79, 3302 (2001).

${ }^{8}$ F. X. Hu, B. G. Shen, and J. R. Sun, Appl. Phys. Lett. 76, 3460 (2000).

${ }^{9}$ R. Jha, S. K. Singh, A. Kumar, and V. P. S. Awana, J. Magn. Magn. Mater. 324, 2849 (2012).

${ }^{10}$ J. C. Debnath, R. Zeng, J. H. Kim, and S. X. Dou, J. Alloys Compd. 509, 3699 (2011).

${ }^{11}$ M. H. Phan, S. C. Yu, and N. H. Hur, Appl. Phys. Lett. 86, 072504 (2005).

${ }^{12}$ V. P. S. Awana, R. Tripathi, N. Kumar, H. Kishan, G. L. Bhalla, R. Zeng, L. S. S. Chandra, V. Ganesan, and H. U. Habermeier, J. Appl. Phys. 107, 09D723 (2010).
} 
${ }^{13}$ Y.-H. Huang, C.-H. Yan, F. Luo, W. Song, Z.-M. Wang, and C.-S. Liao, Appl. Phys. Lett. 81, 76 (2002).

${ }^{14}$ N. T. Trung, L. Zhang, L. Caron, K. H. Buschow, and E. Brück, Appl. Phys. Lett. 96, 172504 (2010).

${ }^{15}$ C. L. Zhang, D. H. Wang, Q. Q. Cao, Z. D. Han, H. C. Xuan, and Y. W. Du, Appl. Phys. Lett. 93, 122505 (2008).

${ }^{16}$ J. T. Wang, D. S. Wang, C. Chen, O. Nashima, T. Kanomata, H. Mizuseki, and Y. Kawazoe, Appl. Phys. Lett. 89, 262504 (2006).

${ }^{17}$ T. Kanomata, H. Ishigaki, T. Suzuki, H. Yoshida, S. Abe, and T. Kaneko, J. Magn. Magn. Mater. 140, 131 (1995).

${ }^{18}$ I. K. Ghosh, C. J. Lobb, R. L. Greene, S. G. Karabashev, D. A. Shulyatev, A. A. Arsenov, and Y. Mukovskii, Phys. Rev. Lett. 81, 4740 (1998).

${ }^{19}$ J. Y. Fan, L. S. Ling, B. Hong, L. Zhang, L. Pi, and Y. H. Zhang, Phys. Rev. B 81, 144426 (2010).

${ }^{20}$ R. Cabassi, F. Bolzoni, A. Gauzzi, and F. Licci, Phys. Rev. B 74, 184425 (2006).

${ }^{21}$ A. Arrott and J. E. Noakes, Phys. Rev. Lett. 19, 786 (1967).

${ }^{22}$ M. Sahana, U. K. Rössler, N. Ghosh, S. Elizabeth, H. L. Bhat, K. Dörr, D. Eckert, M. Wolf, and K. H. Müller, Phys. Rev. B 68, 144408 (2003)
${ }^{23}$ Ch. V. Mohan, M. Seeger, H. Kronmüller, P. Murugaraj, and J. Maier, J. Magn. Magn. Mater. 183, 348 (1998).

${ }^{24}$ H. S. Shin, J. E. Lee, Y. S. Nam, H. L. Ju, and C. W. Park, Solid State Commun. 118, 377 (2001).

${ }^{25}$ J. S. Kouvel and M. E. Fisher, Phys. Rev. 136, A1626 (1964).

${ }^{26}$ S. N. Kaul, J. Magn. Magn. Mater. 53, 5 (1985).

${ }^{27}$ H. E. Stanley, Introduction to Phase Transitions and Critical Phenomena (Oxford University Press, London, 1971).

${ }^{28}$ B. K. Banerjee, Phys. Lett. 12, 16 (1964).

${ }^{29}$ B. Widom, J. Chem. Phys. 41, 1633 (1964).

${ }^{30}$ M. E. Fisher, S. K. Ma, and B. G. Nickel, Phys. Rev. Lett. 29, 917 (1972).

${ }^{31}$ H. Oesterreicher and F. T. Parker, J. Appl. Phys. 55, 4334 (1984).

${ }^{32}$ V. Franco, A. Conde, J. M. Romero-Enrique, and J. S. Blazquez, J. Phys.: Condens. Matter 20, 285207 (2008).

${ }^{33}$ V. Franco, J. S. Blazquez, and A. Conde, Appl. Phys. Lett. 89, 222512 (2006).

${ }^{34}$ M. Halder, S. M. Yusuf, M. D. Mukadam, and K. Shashikala, Phys. Rev. B 81, 174402 (2010).

${ }^{35}$ J. Fan, L. Ling, B. Hong, L. Pi, and Y. Zhang, J. Magn. Magn. Mater. 321, 2838 (2009). 